\title{
Review of antibiotic use in crops, associated risk of antimicrobial resistance and research gaps
}

Area of research interest: Antimicrobial resistance

Study duration: 2019-09-01

Project status: Completed

Project code: FS301082

Authors: Edward Haynes, Carmel Ramwell, Tom Griffiths, Don Walker, Julian Smith

Conducted by: Fera Science Ltd

Date published: 18 November 2020

DOI: https://doi.org/10.46756/sci.fsa.vnq132

\section{Background}

To date, most work on AMR in the agriculture sector has focused on livestock, as animals are directly treated with antibiotics (e.g. to treat infectious disease). It is possible that AMR bacteria and antimicrobial resistance genes can then be transferred to consumers through food.

Crop plants treated with antibiotics represent an alternative potential pathway for bacteria and genes to enter the human microbiome that has been less well recognised. The purpose of this desk study was to summarise the most recent available knowledge on the extent of antibiotic use on crops globally, to indicate the nature of risk that may be associated with these practices, and to set out recommendations and researchable evidence gaps for the UK Government (FSA and Defra) to consider.

\section{Objectives and approach}

Thematic areas of consideration for this project fell into the broad categories of:

- what is known regarding the use of antibiotics to control crop disease, including antibiotic and AMR prevalence in crops;

- what detection methods exist for antibiotic residues and AMR bacteria and genes on crops;

- what is the evidence for the effect of other agrichemicals on AMR;

- associated research gaps.

The commissioned study team conducted a scientific literature survey and attempted to supplement this by expert elicitation through International Plant Protection Covention contact points. However, this International outreach was met with limited success.

Research report

PDF

View Review of antibiotic use in crops as PDF(Open in a new window) (828.19 KB) 